of a possible receptor role of HLA antigens or the "molecular mimicry" between viruses and HLA antigens. Vladutiu (Lancet, ii, 288; 1974) pointed out, however, that lymphocyte-defined determinants have been shown to exhibit a stronger association with diseases than serologically-defined determinants. $\mathrm{He}$ also noted that the enthusiasm generated by the idea of association of HLA antigens with various diseases is not unanimous, and although there is a strong association with a few diseases, the underlying mechanism remains unknown. Vladutiu stressed that there are many pitfalls when searching for such associations and that the principal source of errors is the statistical analysis. Another example cited is the association of many diseases with HLA 1 and HLA 8. Although these antigens are absent in Japanese, diseases known to be associated with them are seen among the Japanese population. It is apparent, therefore, that further studies and experimental models are necessary.

Finally, it is widely recognised that family clustering may be entirely a function of an increased risk of exposure to an infecting virus rather than genetically determined susceptibility; particularly if a parent is excreting the virus and more so if the mother is the transmitter. Mazzur notes that persistent infection with hepatitis $\mathbf{B}$ virus may be representative of other infections which may also mimic genetic patterns. Indeed she suggests that perhaps polygenic birth defects and other diseases, which the currently accepted as genetically determined because of family clustering may also be caused by perhaps as yet unrecognised infectious agents. This suggestion merits considerable further investigation and careful evaluation of the results.

\title{
Theileria in the laboratory
}

from F. E. G. Cox

THE increasing problem of protein shortage in Africa has once again concentrated attention on the parasitic diseases of cattle and although recent attempts at vaccinating animals against these diseases give grounds for optimism (Nature, 260, 380; 1976) much basic research still remains to be done. East Coast Fever, caused by a protozoan Theileria parva and transmitted by a tick Rhipicephalus appendiculatus, still kills up to $40 \%$ of calves over a wide area of Central and East Africa. The parasite develops through two cycles of multiplication in lymphocytes before entering red blood cells and becoming infective to the tick. Only cattle, buffalo and Indian water buffalo can support the infection through to its final infective stages and research has been limited by the non-availability of a suitable laboratory host. In 1970 some progress in the maintenance of $T$. parva in vitro was made (Malmquist, Nyindo and Brown, Trop. Anim. Hlth Prod., 2, 139; 1970) when it was shown that the early stages of the parasite in lymphocytes could be grown in a bovine cell line but the infection did not proceed through to the blood forms. More recently the use of interspecific heterokaryons formed by the virus-induced fusion of cells has found favour (Irvin et al., Int. J. Parasitol., 4 , 519 ; 1974) but still no invasion of blood cells has been recorded.

In this issue of Nature (page 311) Danskin and Wilde describe how they have been able to grow all stages of $T$. parva up to the blood forms in culture. Their technique is simple; they use the modified Eagle's medium described by Malmquist et al. with 20-30\% foetal calf serum (instead of 10\%) and in addition $5-10 \%$ bovine lymph. The usual macroschizonts and microschizonts developed and bovine blood cells added after $18-66 \mathrm{~h}$ became infected but not those added after $90 \mathrm{~h}$. The number of cells infected, $0.1 \%$, was low but cattle are infective to ticks at this level of parasitaemia.

The next stage will be to infect ticks from cultures and considerable progress has been made on an understanding of the feeding biology of $R$. appendiculatus by Tukahirwa (Parasitology, 72, 65; 1976). The larvae and nymphs require $8-9 \mathrm{~d}$ after hatching or moulting before they can feed and this they do for 4-6 d. The adults cannot feed for 6-9 d after moulting but eventually feed for 7-9 d. On Theileria lawrenci (a parasite closely related to $T$. parva, so realistic comparisons are possible) adult $R$. appendiculatus infected as nymphs become infective to cattle $3 \mathrm{~d}$ after beginning to feed and remain so for the next $6 \mathrm{~d}$ (Young et al., Parasitology, 71, 27; 1975). These authors were able to correlate infectivity with morphological criteria of parasite maturity in the ticks' salivary glands so it should be possible to infect nymphs from cultures, allow the nymphs to moult, let them feed on rabbits and collect infective stages to initiate further cultures after $3-4 \mathrm{~d}$ using the morphology of the parasites as a guide to infectivity. Experimental cattle should no longer be needed and basic research will not necessarily have to be done in Africa. This should please the growing number of scientists who are concerned about excessive animal experimentation.

Diseases caused by Theileria are not confined to Africa and in India considerable progress is being made in the understanding of the biology of $T$. annulata. Bhattacharyulu, Chaudri and Gill (Parasitology, 71, 1; 1975) have confirmed that Hyalomma anatolicum and other ticks transmit this disease, that the infection passes from larvae to nymphs and nymphs to adults and that the infection is transmitted during the first $24 \mathrm{~h}$ after feeding. Of considerable interest is the finding that if infected larvae are fed on cattle as nymphs the adults are not infective whereas if the nymphs are fed on rabbits which are not susceptible to infection with $T$. annulata the adults are infective. It seems as if the infective stages are "used up" during feeding on an appropriate host. This may call for some reappraisal of the experimental procedure of using "susceptible" and "nonsusceptible" hosts almost randomly in various fields of research into piroplasmosis. The use of cultured parasites would avoid this problem.

\section{Of gods and men}

\section{from David W. Hughes}

STARs were first named by the herdsmen, hunters, sailors and travellers who lived along the banks of the Euphrates and Ganges, and it was these people and not the learned and scientific who were the sideronomists. The star names used today have been gradually accumulated over the last three thousand years or so. They have come in the main from Greece, Rome and the nomadic and scholarly tribes of Arabia. Castor and Pollux ( $\alpha$ and $\beta$ Geminorum)-the heavenly twins; Regulus ( $\alpha$ Leonis) - the ruler of the affairs of the heavens; Betelgeuze ( $\beta$ Orionis) from ibt al jauzah, the armpit of the central one, Algol ( $\beta$ Persei) from the Arabian's Ra's al Ghul, the Demons head, are a few examples.

Today naming stars has gone out of fashion and astronomers make do with rather dry titles such as V1057 and L789-6. However naming say, Jovian satellites and the features of planetary surfaces that have recently been revealed by imaging devices on space craft is very much in the news and a subject of some controversy.

Carl Sagan (Laboratory for Planetary Studies, Cornell University) discusses this problem in his recent paper in Icarus $(27,575 ; 1976)$. In 1967 , con- 